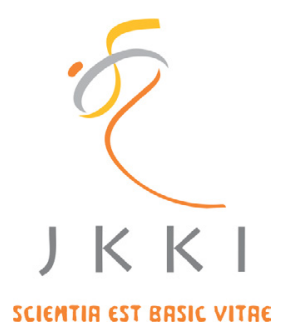

Jurnal Kedokteran dan Kesehatan Indonesia

Indonesian Journal of Medicine and Health

Journal homepage : https://journal.uii.ac.id/JKKI

\title{
Biobank ensuring sustainability in health research
}

Jajah Fachiroh*1,2

${ }^{1}$ Department of Histology and Cell Biology, Faculty of Medicine, Public Health and Nursing Universitas Gadjah

Mada, Yogyakarta, Indonesia

${ }^{2}$ Biobank Development Team , Faculty of Medicine, Public Health and Nursing Universitas Gadjah Mada, Yogyakarta, Indonesia

\begin{tabular}{l}
\hline ART I C L E I NF O \\
*Corresponding author: \\
jajahfachiroh@ugm.ac.id \\
\hline DOI:10.20885/JKKI.Vol11.Iss1.art2 \\
\hline Copyright @2020 Authors. \\
This is an open access article \\
distributed under the terms \\
of the Creative Commons At- \\
tribution-NonCommercial 4.0 \\
International Licence (http:// \\
creativecommons.org/licences/ \\
by-nc/4.0/).
\end{tabular}

\section{EDITORIAL}

$\mathrm{U}$ $\mathrm{K}$ Biobank recruits 500,000 participants of UK inhabitant aged between 40-69 years in 2006-2010 and follow them up longitudinally. UK Biobank collects clinical information, along with biological samples aiming for improving the prevention, diagnosis and treatment of a wide range of serious and life-threatening illnesses including cancer, heart diseases, stroke, diabetes, arthritis, osteoporosis, eye disorders, depression and forms of dementia. ${ }^{1}$ This is a national and international efforts on which the data can already be used by anyone. Paper published until January 2020 is 1,044 and incrementing fast.

The China Kadoorie Biobank aimed to investigate the main genetic and environmental causes of common chronic diseases in the Chinese population during 2004-2008. They have collected around 500,000 extensive data and biosamples. The subjects are further monitored through health registry and insurance links. ${ }^{2}$ Further sustaining researches and publications useful for public health solutions.

Among microbiologists, the American Type Culture Collection (ATCC) providing state of the art reagents, reference microorganisms and cell lines for research and diagnostic purposes. ${ }^{3}$ In the wake of outbreaks, such as Ebola in West Africa 2014-2016 national and international efforts were done to ensure the correct and safe data and biosamples collection, thus the timely collection may provide correct informations shall there is similar outbreaks occur. ${ }^{4}$ Further, driven by development of bioinformatics and collection of large dataset ("big-data") digital pathology uses by the Association of Pathologist in Netherlands has become the first in the world to share pathological images for remote consultations. This modality may become resource for research purpose. ${ }^{5}$ These examples are few of exhaustive biobank formats in sustaining the wheel-of-research, appropriate health service, development of precision medicine and medical technology.

The term "Biobank" was first appeared in Pubmed database in a 1996 paper investigating the role of oxidative DNA damage as an independent risk factor in cancer. ${ }^{6}$ By year 2000 s the publication related to biobank increased dramatically. "Time" magazine in 2009 has identified biobank as one of the "10 ideas that change the world rigt now". Emphasizing the growing functionalities of biobank in clinical service and research aswell. Biobank functions to collect, catalogue and store biological samples and their associated clinical data from a population or fraction of certain population, projecting for long period of time. Biobanks also act as data (and biosamples) stewards, thus responsible for subjects' privacy and ensuring everything related to the subjetcs are used only for intended purposes. From that poin of view, biobank as research (or service) infrastructure becomes a complex science far beyond the "collection of ultrafreezers or liquid nitrogen tanks", connecting technical and non technical aspects.

Scientists often want to collaborate with each other by using existing biosample collections by forming a consortium to reach good statistical power. But then realizing that the biosamples and data 
were collected differently; thus limit the optimalization of data/biosamples use. When in place, biobank may reduce the variability of collection, processing and storage, as biobanks must work in certain standard to prevent false analytical results interpreted as valid findings. Standard operating procedures (SOP), management standards, and quality controls (QC) are commonly applied in clinical laboratories, but hardly in research laboratories. In current years, for biosamples collection purposes, many large laboratories (and those that have operated for long period of time) issue "best practises" covering technical aspects of biosamples collection, including transport, process, storage, quality control, data collection bioinformatics system, standard managements, as well as policy, ethical and legal issues. ${ }^{7}$ These best practises are largely provided online mostly for free, and can be adapted by biobanks alike. Hovewer, international biobanks collaboration requires common standard applied, producing ISO 20387:2018 on biotechnology-biobanking-general requirements for biobanking, for biobanks of multicellular and microbiological biobanking unrelated to food or feed. This ISO standard values the competence and impartiality of biobanks. The application of common standard enables connections among different biobanks and in line with value uses by biobank 3.0 that does not emphasize on the quantity of collections, but to reliability of data ("quality"), stakeholders involvement and sustainability.

To establish and run biobank properly it has to be realized the complexity of biobank that includes different stakeholders to operate. These include stakeholders that provide data (e.g. subjects, biologists, bioinformaticians, clinicians), use the data (e.g. researchers, health service providers), determine norms (e.g. ethicians, community leaders), legal regulation (e.g. lawyers, government, insitutions), and provide fundings (e.g. government, universities, hospitals, philathropies). Thus the opening of a biobank must consider the sustainability plan ensuring long-term service. The biobank sustainability plan must include 1) operational sustainability, aiming to develop policies and procedures to improve the quality of biospecimens and the efficiency of biobank operations; 2) social sustainability, aiming to receive positive perception of the biobank with the public and the researchers it serves; 3 ) financial sustainability, aiming to optimize the ability of the repository to be financially sustainable. ${ }^{8}$

In Indonesia, academic institutions is one of health research champion. Commonly, academic-based biosample collections are characterized by principal investigators' research interest, often hampered by limited support and inconsistent funding. ${ }^{9}$ However, when managed well in standardized protocol, this specific collections may established into disease-specific biobank. Different institutions may establish different type biobank driven by needs and available resources. For instance, biobank developed by department of Pathology Anatomy FK-UI, increased the functionality of "leftover" diagnostic tissues into well-identified biosample resources for cancer-related studies. Biobank at Faculty of Medicine, Public Health and Nursing Universitas Gadjah Mada focus its service for storage of biosamples and their parallel data in structured and standardised manner, while encouraging researchers to collaborate by common utilization of biosamples collected/owned by those who uses biobanks' facility.

Despite the complexity to develop and run biobank, potentials owned by different research interests, institutions, ethnical backgrounds and specific health problems should be the driving force to develop institutional, regional or national biobank in Indonesia. Research-based national health policy requires solid evidence. Initiatives including setting up Indonesian Biobank Network for Health Research has been done for the last 5 years. The initiative is marked by yearly workshop to harmonize understanding and capacity among "biobankers". Continued discussions with stakeholders, and establishing studies on biobank models. ${ }^{10}$ are several evidence of the growing interest on biobank development in Indonesia. Supports from the policy-maker related to the legal aspect of biobank for health research, policy on data ownership and datasharing, as well as the ongoing discussion of ethical concerns on broad and longterm data use needs support from scientific community. Remember, think big (and smart), start small (and persistent).

\section{Acknowledgement}

Thank you to Tri Rini Nuringtyas for reading the draft and your valuable suggestion.

\section{REFERENCES}

1. Conroy M, Sellors J, Effingham M, Littlejohns TJ, Boultwood C, Gillions L, et al. The advantages of UK Biobank's open-access strategy for health research. Journal of Internal Medicine. 
2019;286(4):389-97.

2. Chen Z, Lee L, Chen J, Collins R, Wu F, Guo Y, et al. Cohort profile: The Kadoorie Study of Chronic Disease in China (KSCDC). International Journal of Epidemiology. 2005;34(6):1243-9.

3. De Paoli P. Biobanking in microbiology: From sample collection to epidemiology, diagnosis and research. FEMS Microbiology Reviews. 2005;29(5):897-910.

4. Abayomi A, Katz R, Spence S, Conton B, Gevao SM. Managing dangerous pathogens: challenges in the wake of the recent West African Ebola outbreak. Global Security: Health, Science and Policy. 2016;1(1):51-7.

5. van Diest PJ, Huisman A, van Ekris J, Meijer J, Willems S, Hofhuis $\mathrm{H}$, et al. Pathology Image Exchange: The Dutch Digital Pathology Platform for Exchange of Whole-Slide Images for Efficient Teleconsultation, Telerevision, and Virtual Expert Panels. JCO Clinical Cancer Informatics. 2019;(3):1-7.

6. Loft S, Poulsen HE. Cancer risk and oxidative DNA damage in man. Journal of Molecular Medicine. 1996;74(6):297-312.

7. Vaught J, Lockhart NC. The evolution of biobanking best practices. Clinica Chimica Acta. 2012;413(19-20):1569-75.

8. Watson PH, Nussbeck SY, Carter C, O'donoghue S, Cheah S, Matzke LAM, et al. A framework for biobank sustainability. Biopreservation and Biobanking. 2014;12(1):60-8.

9. Uzarski D, Burke J, Turner B, Vroom J, Short N. A Plan for Academic Biobank Solvency-Leveraging Resources and Applying Business Processes to Improve Sustainability. Clinical and Translational Science. 2015;8(5):553-7.

10. Fachiroh J, Dwianingsih EK, Wahdi AE, Pramatasari FLT, Hariyanto S, Pastiwi N, et al. Development of a biobank from a legacy collection in universitas gadjah mada, Indonesia: Proposed approach for centralized biobank development in low-resource institutions. Biopreservation and Biobanking. 2019;17(5):387-94. 